\title{
Stochastic modeling of near-field exposure to parabens in personal care products
}

\author{
Csiszar, Susan A.; Ernstoff, Alexi; Fantke, Peter; Jolliet, Olivier
}

Published in:

Journal of Exposure Science and Environmental Epidemiology

Link to article, DOI:

$10.1038 /$ jes.2015.85

Publication date:

2017

Document Version

Peer reviewed version

Link back to DTU Orbit

Citation (APA):

Csiszar, S. A., Ernstoff, A., Fantke, P., \& Jolliet, O. (2017). Stochastic modeling of near-field exposure to parabens in personal care products. Journal of Exposure Science and Environmental Epidemiology, 27(2), 152159. https://doi.org/10.1038/jes.2015.85

\section{General rights}

Copyright and moral rights for the publications made accessible in the public portal are retained by the authors and/or other copyright owners and it is a condition of accessing publications that users recognise and abide by the legal requirements associated with these rights.

- Users may download and print one copy of any publication from the public portal for the purpose of private study or research.

- You may not further distribute the material or use it for any profit-making activity or commercial gain

- You may freely distribute the URL identifying the publication in the public portal 
Csiszar SA, Ernstoff AS, Fantke, P, Jolliet O. Stochastic modeling of near-field exposure to parabens in personal care products. Journal of Exposure Science and Environmental Epidemiology. 2016. Available from, DOI: 10.1038/jes.2015.85 Alternative contact email: ojolliet@umich.edu

\section{Stochastic modeling of near-field exposure to parabens in personal care products}

Susan A. Csiszar ${ }^{a^{*}}$ Ph.D., Alexi S. Ernstoff ${ }^{b}$ M.Eng., Peter Fantke ${ }^{b}$ Ph.D., and Olivier Jolliet ${ }^{a}$ Ph.D.

${ }^{a}$ Environmental Health Sciences, School of Public Health, University of Michigan, Ann Arbor, MI, United States

${ }^{\mathrm{b}}$ Quantitative Sustainability Assessment Division, Department of Management Engineering, Technical University of Denmark, Kgs. Lyngby, Denmark

*Corresponding author: 1415 Washington Heights, Ann Arbor, Michigan 48109-2029

Tel: 19377893608 Email: scsiszar@ umich.edu

Running title: Personal care product exposure modeling

Conflict of interest.

The authors declare no conflict of interest.

23 


\section{Abstract}

Exposure assessment is a key step in determining risks to chemicals in consumer goods including personal care products (PCPs). Exposure models can be used to estimate exposures to chemicals in the absence of biomonitoring data and as tools in chemical risk prioritization and screening. We apply a PCP exposure model based on the product intake fraction $(\mathrm{PiF})$, which is defined as the fraction of chemical in a product that is taken in by the exposed population, to estimate chemical intake based on physicochemical properties and PCP usage characteristics. The PiF can be used to estimate route and pathway specific exposures during both the use- and disposalstages of a product. As a case study, we stochastically quantified population level exposures to parabens in PCPs, and compared estimates to biomarker values. We estimated exposure based on the usage of PCPs in the female US population, taking into account population variability, product usage characteristics, paraben occurrence in PCPs, and the PiF. Intakes were converted to urine levels and compared to NHANES (National Health and Nutrition Examination Survey)

37 biomonitoring data. Results suggest that for parabens, chemical exposure during product use is 38 substantially larger than environmentally mediated exposure after product disposal. Modeled urine concentrations reflect well the NHANES variation of three orders of magnitude across parabens for the $50^{\text {th }}, 75^{\text {th }}, 90^{\text {th }}$, and $95^{\text {th }}$ percentiles and were generally in good agreement with measurements, when taking uncertainty into account. This study presents an approach to estimate

42 multi-pathway exposure to chemicals in PCPs and can be used as a tool within exposure based screening of chemicals as well in higher tier exposure estimates.

Keywords: exposure modeling, dermal exposure, inhalation exposure, multi-media studies, 


\section{Introduction}

In order to inform risk assessment of chemicals in cosmetics and personal care products

51 (PCPs) an understanding of individual and population level exposure is required. ${ }^{1,2}$ The need for

52 exposure estimates based on various chemical uses is highlighted by the recent advances in high-

53 throughput exposure models for chemical prioritization ${ }^{3,4}$ which can also be combined with high-

54 throughput toxicity estimates to inform risk. ${ }^{5,6}$ Historically, these modeling efforts have focused

on far-field environmentally mediated exposures and less on near-field pathway exposures

occurring indoors and during product use. ${ }^{3,7}$ Usage of PCPs has been shown to be well correlated

57 with exposure ${ }^{8,9}$ and use-phase exposure has been estimated to be greater than environmentally methods for chemicals in consumer products. ${ }^{6}$

Several calculations have been developed to estimate chemical intake via PCP use and are

62 based on multiplicative models ${ }^{1,2,12}$ using a set skin permeation fraction often derived from the

63 literature and do not necessarily take exposure duration (e.g. rinse-off versus leave-on into

64 account). ${ }^{1}$ On the other hand, models have been developed to estimate the skin permeation

65 coefficient of a chemical ${ }^{13,14}$ and chemical uptake into the skin. ${ }^{15,16}$ Skin permeation models

66 provide the advantage that they can be applied to chemicals based on physicochemical properties

67 (i.e. octanol-water partition coefficient, $K_{o w}$, and molecular weight) thereby lending themselves

to computationally based calculations rather than relying on data from the literature which is not

69 conducive to multiple chemical calculations. Furthermore, models used to estimate exposure to

70 chemicals applied dermally vary in mathematical complexity, for example by assuming only one

71 chemical fate pathway (i.e. dermal uptake), ${ }^{15}$ or only providing complex numerical solutions. ${ }^{16}$ 
72 Modeling frameworks are currently being developed to combine dermal uptake with the concept

73 of the product intake fraction, PiF, defined as the fraction of the chemical in a product that is

74 eventually taken in by the exposed individual(s)/population. ${ }^{17}$ These models can be applied to

75 predict chemical intake via several different pathways such as dermal uptake, inhalation intake

76 and gaseous dermal uptake of volatilized chemicals, and to environmentally mediated exposure

77 after product disposal. The advantage of this multi-pathway approach is that the relative

78 contribution of each pathway can be estimated and does not assume that exposure only occurs

79 via dermal uptake of product applied to the skin and allows for comparison between use-phase

80 and disposal-phase exposures.

Such models, however, have yet to be evaluated on a population level using for example,

82 biomarker data. Additionally, exposure is often estimated based on the usage of a single product rather than an aggregate analysis taking into account usage of multiple products containing a

84 given chemical ${ }^{1,2,15}$, which may underestimate a consumer's entire exposure for chemicals found 85 in multiple product types. Cowan-Ellsberry and Robison ${ }^{1}$ and Gosens et al. ${ }^{2}$ present aggregate 86 exposure estimates for parabens in PCPs, however their estimates do not use a skin permeation

87 model nor are their estimates validated against biomarker data. Delmaar et al. ${ }^{15}$ estimated aggregate exposure to diethyl phthalate using a skin permeation model, however they only

89 consider the dermal exposure pathway. The application of a multi-pathway exposure model to estimate cosmetic intake has not been validated on a population level nor applied across multiple

91 product types to yield aggregate exposure estimates. To address this gap, the product intake

92 fraction concept would need to be adapted to several PCPs and validated against population level data. Evaluation of a PCP exposure model using data rich chemicals will build further 
94 confidence in these techniques such that they can be incorporated into Tier 1 exposure and risk 95 screening approaches and used on a broader range of chemicals. In this paper, we apply the PiF concept to model chemical intake due to PCP usage using

97 parabens as a case study. In order to compare the estimated intakes to population-based biomonitoring data we probabilistically combined the PiF calculations with aggregate exposure considerations to capture population variability, focusing on a class of widely used chemicals. This type of analysis is referred to as a Tier 2 probabilistic exposure estimate ${ }^{18}$ and is more detailed than point estimates often used in screening approaches (Tier 1). ${ }^{6}$ Parabens are 102 commonly used in PCPs and cosmetics as preservatives, are readily absorbed into the skin, ${ }^{19}$ are 103 detectable in urine, ${ }^{20}$ and thereby provide a good PCP exposure case study. Urinary biomarker 104 data is available for the US population from NHANES (National Health and Nutrition 105 Examination Survey) $)^{21}$ where parabens have been detected in $\sim 99 \%$ of the population. ${ }^{22}$ Additionally, parabens are suspected endocrine disruptors ${ }^{23,24}$ and these exposure calculations provide a basis for informing risk when combined with toxicity, bioactivity, or allowable dose data. ${ }^{17}$ This study therefore aims to:

1. Estimate and contrast modeled product intake fraction for various exposure pathways (including near and far-field exposures) for parabens in a variety of PCPs and cosmetics while accounting for both chemical specific properties and product use characteristics. from the usage of multiple PCPs.

3. Evaluate the stochastic method by comparing its predictions with NHANES urine 116 concentrations at different percentiles $\left(50^{\text {th }}\right.$ to $\left.95^{\text {th }}\right)$ of exposure in the US population.

\section{Methods}


We used four common parabens methyl, ethyl, propyl, and butyl paraben (MeP, EtP, PrP,

BuP, respectively) and eleven commonly used personal care products (PCPs) as a case study. We included rinse-off products (shampoo, conditioner, facial cleanser, body wash) and leave-on products (body lotion, face cream, night cream, deodorant, foundation, eye shadow, and lipstick).

We studied the U.S. female population due to the availability of urine biomarker data ${ }^{21}$ and the significantly higher exposure of the female versus male population to parabens. ${ }^{22}$

\subsection{Product Intake Fraction}

We used the product intake fraction $(\mathrm{PiF})$ metric to assess the fraction of parabens in products that humans are exposed to a) during product use and b) via subsequent environmental emissions after product use. The $\mathrm{PiF}$ is defined as the ratio of the amount of chemical in a product that is taken in by humans and the amount of chemical contained in that product and depends on physicochemical properties as well as product use characteristics. ${ }^{17}$ Once a product is applied we assumed that it can undergo the following pathways: direct dermal uptake into the skin, volatilization to air, and washed down-the-drain after the product is rinsed off. Following from these pathways, we calculated a PiF for each of the following exposure pathways: dermal uptake of chemical in an aqueous product $\left(P i F^{\mathrm{derm}, \mathrm{aq}}\right)$, inhalation of chemical from the volatilized product $\left(P i F^{\mathrm{inh}}\right)$, gaseous dermal uptake of chemical from the volatilized product $\left(P i F^{\mathrm{derm}, \mathrm{gas}}\right)$, and environmentally mediated chemical intake due to disposal after product use $\left(P i F^{\text {disp }}\right)$, for each paraben and each PCP type.

The expression for dermal uptake of chemicals in aqueous products into the skin $\left(P i F^{\mathrm{derm}, \mathrm{aq}}\right)$ is based on a two-compartment mass balance between product and skin and yields the following solution (see Table S1 in Supplementary Information (SI)):

$$
P i F^{\text {derm,aq }}=\frac{k_{\mathrm{ps}}}{k_{\mathrm{ps}}+k_{\mathrm{pa}}}\left(1-\mathrm{e}^{-\left(k_{\mathrm{ps}}+k_{\mathrm{pa}}\right) t}\right)
$$


where $k_{\mathrm{ps}}\left(\mathrm{h}^{-1}\right)$ and $k_{\mathrm{pa}}\left(\mathrm{h}^{-1}\right)$ are the product-skin and product-air transfer rates, respectively and

$141 t(\mathrm{~h})$ is the exposure time, that is the duration that the product stays on the skin before being

142 washed-off. The transfer rates $k_{\mathrm{ps}}$ and $k_{\mathrm{pa}}$ are both functions of the thickness of product on the

143 skin, in addition to chemical specific parameters such as the aqueous skin permeation

144 coefficient, $K_{\mathrm{p}}^{\mathrm{aq}}\left(\mathrm{cm} \mathrm{h}^{-1}\right)$ and the air-water partition coefficient $\left(K_{\mathrm{aw}}\right)$ respectively. Expressions for

145 the intake of volatilized chemical via inhalation $\left(P i F^{\mathrm{inh}}\right)$ and gaseous dermal uptake $\left(P i F^{\text {derm,gas }}\right)$

146 are given in SI, Table S1, and are summed with $P i F^{\text {derm,aq }}$ to constitute the total use-stage, $P i F^{\text {use }}$,

147 via these exposure routes.

148 The PiF associated with product disposal, $P i F^{\text {disp }}$ (SI, Table S1), was modeled as the fraction

149 of chemical not taken in during use and subsequently washed down the drain into a waste water

150 treatment plant (WWTP) and then released to environmental compartments (air, water, soil). The

151 subsequent environmental intake fractions (iF) were calculated using the USEtox model. ${ }^{25}$

152 Finally, a chemical and product specific $P i F^{\text {tot }}$ can be defined as the sum of chemical intakes via

153 all considered pathways (i.e., $P i F^{\text {derm,aq }}, P i F^{\text {derm,gas }}, P i F^{\text {inh }}, P i F^{\text {disp }}$ ) and represents the total

154 chemical intake via all exposure routes. More details on the calculation of the various pathway

155 and route specific PiFs can be found in the SI (Section S1).

156 We note that some personal care products may also lead to non-dietary ingestion exposure,

157 for example mouthwash and toothpaste, however these products are not reported to contain

158 parabens ${ }^{12,26}$ and were thus not included in the model. An ingestion PiF can be readily

159 incorporated into this modeling framework and can take the value of the fraction of product that

160 is ingested per product use. For example, for toothpaste Bremmer et al. ${ }^{27}$ used measured values

161 to estimate a toothpaste ingestion fraction. We did not include an ingested fraction for lipstick as 
162 the resulting median $P i F^{\text {derm,aq }}$ was already larger than $50 \%$ for all parabens, and previously

163 assumed fixed ingestion fractions for lipstick can vary greatly (e.g. from $0.1-100 \%){ }^{4,27}$

$164 \quad 2.2$ Total daily intake

165 The $P i F^{\mathrm{tot}}$ can then be used to calculate daily intake for a given chemical in a product and can 166 be summed across several different products (p) to calculate an aggregate chemical intake, $I(\mathrm{mg}$ $\left.167 \mathrm{~kg}^{-1} \mathrm{~d}^{-1}\right)$ as

$168 I=\frac{\sum_{\mathrm{p}} P i F_{\mathrm{p}}^{\mathrm{tot}} M_{\mathrm{p}} f_{\mathrm{p}}}{B W}$

169 where $M_{\mathrm{p}}\left(\mathrm{mg} \mathrm{d}^{-1}\right), f_{p}$, and $B W(\mathrm{~kg})$ are the daily mass of product applied, fraction of chemical 170 in the product, and body weight, respectively.

$171 \quad 2.3$ Monte Carlo Analysis, Model Parameterization, and Aggregate Exposure

172 Several of the parameters used as input to model the intake of chemicals in PCPs are subject

173 to population variability, i.e. can have a range of possible values depending on individual

174 characteristics and behavior within the studied population. We used Monte Carlo (MC) analysis

175 to incorporate this population variability into our intake calculations. The parameters included in 176 the analysis as well as their distributions are listed in SI, Table S5. The MC analysis was carried

177 out for each product and chemical combination by generating $10^{5}$ random values for each input

178 parameter from the given probability distribution and using these values to calculate an intake

179 distribution. Thus in total for four parabens in 11 PCPs yields 44 calculated intake distributions.

180 We note that the MC analysis considered variables to be independent and potential impacts of 181 variable correlations were not assessed.

182 Several key parameters dictate the calculated PiF and intake for a given product-chemical

183 combination and include the aqueous skin permeation coefficient, $K_{\mathrm{p}}^{\mathrm{aq}}$, the daily amount of 
184 product used, $M_{\mathrm{p}}$, and the fraction of chemical in the product, $f_{\mathrm{p}}$ (Eq. 2). We collected

185 empirical values of $K_{\mathrm{p}}^{\mathrm{aq}}$ for parabens conducted in different media such as an aqueous solution 186 or with an added alcohol and based the input distribution on these values ${ }^{28-33}$ (Table 1 and S3).

187 For the daily mass of product usage, we used distribution data from Loretz et al. ${ }^{34-36}$ as these 188 data pertain specifically to the U.S. female population and detailed information on distributions 189 were available for the MC analysis. Generally, there is limited information available on the 190 chemical composition of consumer products, including PCPs. ${ }^{26}$ We collected fraction paraben 191 content information from various sources ${ }^{1,12,37}$ and aggregated this data into a uniform 192 distribution (see SI Section S3 for details) with example values for shampoo and body lotion in 193 Table 1.

194 Up until this point, all calculations were described for a given product-chemical combination 195 used by the exposed population and do not account for the population with zero exposure. In 196 reality, different consumers use different combinations of products, with some products 197 containing paraben(s) and some not. Thus, in order to calculate aggregate exposure to PCPs, the 198 probability of occurrence of a given chemical within a PCP (percentage of products with a given 199 paraben) and the probability of product use (percentage of population that uses a given product) 200 need to be taken into account ${ }^{1}$ (Figure S4, SI). The initial distributions created for the chemical 201 intake of the exposed population are thus adjusted for the unexposed population by adding the 202 appropriate amount of zeros representing non-exposure to the 44 distributions of $10^{5}$ values 203 calculated for the exposed population.

\subsection{Product co-use}

205 To calculate the population exposure to parabens, the co-use of PCPs should also be taken 206 into account. For a given paraben there are eleven intake distributions representing each product 
207 with several entries representing zero exposure based on the exposure probability; we randomly

208 permeated these distributions and then summed intake across products. This yields a single

209 aggregated intake distribution for each paraben (four distributions in total) with each entry

210 representing a random sum of product intake percentiles with some products having zero intakes.

211 We note that paraben exposure can occur via other media such as food and dust, ${ }^{19,38,39}$ however

212 these media have been estimated to contribute substantially less to exposure levels when

213 compared to those occurring from direct PCP use. ${ }^{1,12,39}$

214 To demonstrate the potential usage of the PiF for risk screening, we also applied Eq. (2)

215 using the $99^{\text {th }}$ percentiles for all values (except body weight, which was set to a constant $75 \mathrm{~kg}$ )

216 and added intake across all eleven products to yield a high-end usage scenario. This intake

217 estimate represents a user who uses all eleven products which all contain parabens, and does not

218 take into account any of the exposure adjustments described above. This intends to represent the

219 very high-end of potential exposure, within the intended use of PCPs.

\subsection{Converting external intake into urine concentrations}

221 The inclusion of the MC analysis to produce intake distributions also allows for comparison

222 to NHANES biomonitoring data which is in the form of population percentiles. ${ }^{21}$ In order to

223 compare modeled intakes to biomonitoring data, we converted the dose taken in into urine

224 concentrations based on the urinary excretion fraction, $f_{\mathrm{UE}}$, of the chemical. Following Angerer

225 et al. ${ }^{40}$ the creatinine corrected chemical content in urine, $C_{\mathrm{Cr}}\left(\mathrm{mg}_{\text {intake }} \mathrm{mg}_{\text {excreted }}^{-1}\right)$, can be

226 estimated as

$227 \quad C_{\mathrm{Cr}}=\frac{I \times B W \times f_{\mathrm{UE}}}{C r_{24 \mathrm{~h}}}$ 
228 where $C r_{24 h}\left(\mathrm{mg}_{\text {excreted }} \mathrm{d}^{-1}\right)$ is the daily creatinine excretion rate. There is very limited data on

$229 f_{\mathrm{UE}}$ for parabens available in the literature. ${ }^{41}$ Thus, we estimated $f_{\mathrm{UE}}$ values based on measured

230 in vitro renal and hepatic clearance rates. ${ }^{42,43}$ Uncertainty in $f_{\mathrm{UE}}$ was included in the analysis by

231 setting upper and lower bounds and running two sets of MC calculations using these high and

232 low bounds of $f_{\mathrm{UE}}$ (see SI, Section S5). We also added $f_{\mathrm{UE}}$ and $C r_{24 h}$ from Eq. (3) to the Monte

233 Carlo analysis to account for population variability in these parameters. For further details on

$234 f_{\mathrm{UE}}$ see SI, Section S5. Eq. (3) was applied to each of the four paraben aggregate intake

235 distributions to yield distributions for paraben urinary concentrations. The percentiles from these

236 distributions can then be compared to the population based urinary concentration percentiles

237 available from NHANES $\left(50^{\text {th }}, 75^{\text {th }}, 90^{\text {th }}\right.$, and $95^{\text {th }}$ percentiles for the years $\left.2009-2010\right) .^{21}$

238 3. Results

2393.1 Paraben Product Intake Fraction

240 The median product intake fraction during the use-stage, $P i F^{\text {use }}$ ranged from $2-88 \%\left(2.5^{\text {th }}\right.$ -

$24197.5^{\text {th }}$ percentiles ranged from $0.1-99 \%$ ) across the product-chemical combinations, with the

242 highest $P i F^{\mathrm{use}}$ for EtP in body lotion and the lowest for EtP in conditioner (Figure 1). This

243 indicates that a substantial fraction of the parabens in cosmetics penetrates the skin (Figure S2).

244 In contrast, the mean environmentally mediated $P i F^{\text {disp }}$ was three to four orders of magnitude

245 lower than $P i F^{\text {use }}$ for all product-chemical combinations, ranging from $10^{-4}-10^{-3} \%$ with the

246 highest $P i F^{\mathrm{disp}}$ for PrP in shampoo and the lowest for EtP in body lotion. This implies that the

247 focus can be on the use-stage exposure, which is substantially higher than environmentally

248 mediated exposure, such that the disposal-stage was subsequently excluded from the Monte

249 Carlo analysis. Within the use-stage, dermal aqueous uptake accounted for 78-99\% of the total 
250

251

252

253

254

255

256

257

258

259

260

261

262

263

264

265

266

267

268

269

270

271

272

$\mathrm{PiF}^{\mathrm{use}}$ indicating that exposure to parabens in PCPs is dominated by direct dermal intake of chemical applied to the skin (Figure S2). As the second main impact pathway, gaseous dermal uptake accounted for 1 to $21 \%$ of $P i F^{\mathrm{use}}$ and inhalation represented only 0.1 to $1 \%$ of $P i F^{\mathrm{use}}$. Weschler and Nazaroff ${ }^{44}$ also found that dermal gaseous uptake exceeds the inhalation pathway for parabens.

The large range in $P i F^{\mathrm{use}}$ for parabens is mostly due to the variation in the application duration of each PCP; $P i F^{\mathrm{use}}$ ranged from 6-50\% for rinse-off products with a mean application duration of 4 minutes, and from $50-80 \%$ for leave-on products with a mean application duration of 14 hours. A plot of $P i F^{\text {derm,aq }}$ versus time (Figure S3a) for the mean product thickness, $h(0.01$ $\mathrm{cm})$, shows that at 4 minutes $(0.07 \mathrm{~h})$, chemical uptake is still in the linear phase of the exponential (with $P i F^{\text {derm,aq }}$ ranging from $0.2-0.4$ ), whereas at 14 hours, uptake has reached its plateau. For parabens this plateau occurs for $P i F^{\text {derm,aq }}$ at or above $80 \%$ at the mean product thickness indicating that parabens are readily absorbed into the skin, which has been observed empirically, ${ }^{32,45}$ whereas this plateau may occur at a substantially lower $P i F^{\text {derm,aq }}$ for more volatile chemicals. This observation is in-line with Gouin et al. ${ }^{46}$ who suggested that wash-off products (as opposed to leave-on products) are likely the dominant source of PCP chemicals to WWTPs, noting that this depends on physicochemical properties. For a given chemical, increasing product thickness can also reduce the fractional aqueous uptake (although not necessarily the overall intake via this pathway); thus body wash (mean $h=0.003 \mathrm{~cm}$ ) had a larger $P i F^{\text {derm,aq }}$ than shampoo (mean $h=0.03 \mathrm{~cm}$ ) (Figure 1) while both are rinse-off products (this is demonstrated in a plot of $P i F^{\text {derm,aq }}$ versus time using the product thickness for body wash and shampoo (Figure S3b)). Overall, mean product thicknesses ranged from $10^{-4}$ (body lotion) to $10^{-2}$ (shampoo) and are a function of the surface area of the application area and amount of 
273 product applied (Table S1). The $P i F^{\text {derm,aq }}$ was more sensitive to product thickness for rinse-off

274 products as the uptake plateau is reached at 8 hours irrespective of leave-on product thickness

275 (Figure S3b).

$276 \quad 3.2$ Population level paraben intakes

277 Figure 2 presents the relationship between the potential doses of chemical used (assuming

$278100 \%$ product usage in the population and $100 \%$ paraben occurrence) and the effective chemical

279 intake after the indicated adjustment (i.e. product usage, paraben occurrence, and $\mathrm{PiF}$ ) to the

280 previous adjustment. Adjusting for product usage reduced the potential dose by a factor of 1.4

281 for the four parabens on average, and the subsequent adjustment for paraben occurrence reduced

282 the potential dose by a factor 1.5 for MeP up to a factor 6 for BuP. Multiplying the effectively

283 applied dose (i.e. after adjusting for product usage and occurrence) by the PiF reduced the

284 population exposure by 3, 1.5, 4, and 2 times for MeP, EtP, PrP, and BuP, respectively. Overall,

285 the final adjusted intakes were 7, 11,13 and 20 times lower than the potential dose for MeP, EtP,

286 PrP, and BuP, respectively. Cowan-Ellsberry and Robison ${ }^{1}$ also found that applying these

287 refinements substantially reduced the population exposure of parabens in PCPs with reductions

288 ranging from a factor 2 to 12.5 . Using PrP as an example, body wash, shampoo, body lotion, and

289 conditioner contributed most to the potential applied dose $(26,22,18$, and $18 \%)$. Once the

290 refinements were applied, body lotion and body wash dominated the total intake (38\% and $28 \%$,

291 respectively), whereas shampoo and conditioner combined made up only $13 \%$ of the total intake

292 due to lower exposure duration and PiF (Figure 2).

293 Accounting for product co-use yielded the final modeled exposure distributions for the four

294 parabens (Figure 3). Based on these distributions $\sim 100,75,97$, and $69 \%$ of the adult female

295 population is exposed (i.e. with non-zero intakes) to MeP, EtP, PrP, and BuP, respectively which 
compares well to $99,42,93$, and $47 \%$ detection reported for all urine samples (i.e. representing the entire population) from NHANES ${ }^{22}$ (Figure S5, SI). The higher detection frequency we determined for female adults are consistent with the highest NHANES paraben urine concentrations for female adults compared to other population groups. MeP and PrP had the highest probability of exposure out of the four parabens (Figure 3), due to their higher frequency of occurrence, while the modeled EtP and BuP intakes were strongly reduced when considering occurrence (Figure 3).

The mean $\left(2.5^{\text {th }}-97.5^{\text {th }}\right.$ percentile $)$ modeled population intakes were $0.2\left(3 \times 10^{-3}-0.8\right), 0.03(0-$ 0.2), $0.06(0-0.3), 0.02(0-0.1) \mathrm{mg} \mathrm{kg}^{-1} \mathrm{~d}^{-1}$ for MeP, EtP, PrP, and BuP, respectively (Table 2). These modeled mean intakes fall in-between those found by Cowan-Ellsberry and Robison ${ }^{1}$ and Guo and Kannan ${ }^{12}$ for paraben exposure due to PCPs (Table S9), noting that these studies did not take population variability into account.

Since some consumers may indeed use all PCP types which may all contain a given paraben, we calculated a high-end intake without applying the exposure adjustments for population exposure (i.e. we did not adjust for product usage, paraben occurrence, and co-use), yielding doses of $8,3,4$, and $2 \mathrm{mg} \mathrm{kg}^{-1} \mathrm{~d}^{-1}$ for MeP, EtP, PrP, and BuP respectively and are approximately an order of magnitude larger than the $99^{\text {th }}$ percentile stochastically based adjusted exposure estimates. While these high-end estimates of exposures may not necessarily be likely, they may be possible and provide upper end conservative exposure estimates.

\subsection{Conversion to biomonitoring levels and comparison to NHANES}

Combining urinary excretion rates with the modeled intakes (with all adjustments, i.e. product usage, paraben occurrence, and $P i F^{\text {tot }}$ ) allows for conversion to urinary concentration distributions, which can be directly compared to the $50^{\text {th }}, 75^{\text {th }}, 90^{\text {th }}$, and $95^{\text {th }}$ percentiles of the 
NHANES biomonitoring data. Modeled urine concentration percentiles reflect well the NHANES variation of three orders of magnitude across parabens and percentiles and were well correlated $\left(\mathrm{R}^{2}=0.9\right.$ comparing the $\left.\log \right)$. Modeled values were within a factor of three (except for one value) using the in vitro estimated values of $f_{\mathrm{UE}}$. When taking uncertainty into account, all modeled values were in agreement with NHANES values (Figure 4). As discussed above, the effect of applying the $P i F^{\mathrm{tot}}$ reduced the product usage and paraben occurrence adjusted intakes on average by a factor of 1.5 to 4 (Figure 2 ) which is a reflection of the median $P i F^{\text {tot }}$ being larger than $50 \%$ for the majority of the products (i.e the leave-on products). This indicates that the three orders of magnitude variation in the biomonitoring data is not only a function of the PiF but also population variability and the other included exposure adjustments. The comparison to biomonitoring data suggests that the estimated PiFs for parabens are within an order of magnitude of actual intake fractions.

\section{Discussion}

The $\mathrm{PiF}$ is a useful metric to compare product specific chemical intake due to various near- and far-field exposure pathways and routes due to PCP use, and for differentiating exposure between leave-on and rinse-off products instead of assuming a fixed fraction of chemical absorbed into the skin, which may lead to overestimates of the exposure. For parabens, model results suggested that dermal aqueous and gaseous uptake were the dominant exposure pathways and the inhalation and far-field pathways were substantially lower in comparison. Gouin et al. (2013) suggested that the use-phase of PCPs may be used to estimate down-the-drain emissions of PCP chemicals and the framework presented here can also be applied in this context. Furthermore, the PiF for PCPs has an analytical solution and can be calculated based on physicochemical properties and product usage characteristics and thus lends itself to rapid 
342 computational exposure estimates. While this study applied stochastic techniques in-line with

343 Tier 2 exposure calculations ${ }^{18}$ to facilitate comparison with bioactivity data, the PiF modeling

344 framework for PCPs can also be readily applied in Tier 1 screening assessments as recommended

345 by Shin et al. ${ }^{6}$

Other exposure media for parabens include food and dust ${ }^{19,38,39}$, however these sources

347 have been previously found substantially lower than PCPs. ${ }^{1,12,39}$ Soni et al. ${ }^{19}$ estimated that the

348 highest likely food intake for MeP and PrP is $0.01 \mathrm{mg} \mathrm{kg}^{-1} \mathrm{~d}^{-1}\left(1 \mathrm{mg} \mathrm{d}^{-1}\right.$ normalized to $75 \mathrm{~kg}$ used

349 in this study), which is 20 and 6 times lower than our modeled mean intake due to PCP usage for

$350 \mathrm{MeP}$ and PrP, respectively. The $95^{\text {th }}$ percentile food intake for the four parabens ranged from $10^{-}$

$351 \quad{ }^{4}-10^{-6} \mathrm{mg} \mathrm{kg}^{-1} \mathrm{~d}^{-1}$ based on measured food concentrations of parabens in U.S. ${ }^{39}$ Intake of the four

352 parabens via dust based on measured dust concentrations was estimated to range between $10^{-6}$ -

$35310^{-9} \mathrm{mg} \mathrm{kg}^{-1} \mathrm{~d}^{-138}$ and is several orders of magnitude lower than modeled PCP intakes.

354 To predict paraben intake accurately, it is crucial to account for product usage, paraben

355 occurrence within products, and population variability. Accounting for these exposure

356 adjustments and using modeled PiF for parabens in PCPs yielded agreement between modeled

357 and NHANES urine concentrations. This indicates that a detailed exposure calculation taking

358 into account the chemical and product dependent $\mathrm{PiF}$, exposure probability, and population

359 variability can be an effective method to predict population level chemical intake associated with 360 PCPs.

361 Uncertainty on the fraction urinary excretion, $f_{\mathrm{UE}}$, is considerable when converting

362 chemical intakes and the limited empirical data available in the literature for parabens resulted in 363 high uncertainty in estimated urine concentrations. Physicochemical property based estimates of 364 pharmacokinetic parameters have recently been made available ${ }^{42,47}$ and may be useful for 
comparing exposure with high-throughput toxicity data for a larger number of chemicals. While the mean modeled urine levels overestimate those of NHANES the $\mathrm{R}^{2}$ of 0.87 for the $\log$ fit of modeled versus measured indicates that the modeling approach presented here was able to capture the exposure patterns of the four parabens well.

An additional challenge when estimating aggregate exposure is to effectively take into account product co-use. ${ }^{1}$ While several PCP usage studies report some data on product co-use, this information cannot be practically applied to a comprehensive PCP study as the data presentation is often incomplete; for example, only the most commonly used combinations, the correlation between the use of two products (rather than multiple products), or data on different sets of PCPs are presented, ${ }^{1,48,49}$ and certain PCPs of interest are not included in that dataset. By accounting for the probability of using a product, we were able to provide an initial reasonable estimate of product co-use, which could be complemented by multiple product usage conditional probabilities. Furthermore, co-use becomes even more complex when chemicals occur in different product types, for example PCPs and cleaning products and alternative methods for taking co-use into account may be needed.

Within the context of risk screening, the exposure refinements needed for a population level calculation may not necessarily be needed to calculate exposure for high-end product users (for example, those who use several PCPs with high-end product masses within the intended product usage) to protect all users rather than an average user. For example, exposure estimates can be compared with the allowable daily intake (ADI) to inform risk of parabens in PCPs. While there is no ADI for EtP and BuP, the combined ADI for MeP and PrP in the European Union is $0-10 \mathrm{mg} \mathrm{kg}^{-1} \mathrm{~d}^{-1} 19,23$ which is within an order of magnitude of both the high-end user 
387 combined intake of $12 \mathrm{mg} \mathrm{kg}^{-1} \mathrm{~d}^{-1}$ and the $99^{\text {th }}$ percentile stochastically estimated intake (with 388 exposure adjustments) of $1.5 \mathrm{mg} \mathrm{kg}^{-1} \mathrm{~d}^{-1}$.

389 We presented a detailed population level PCP exposure model which is able to predict the 390 three orders of magnitude of variation in NHANES paraben urine concentrations. The PCP

391 product intake fraction model can be readily incorporated into rapid exposure models and can be 392 combined with concentration databases such as the recently released Consumer Product 393 Chemical Profile database $\mathrm{CPCPdb}^{26}$ to estimate chemical intakes due to PCP use.

\section{Acknowledgements}

397 We thank Barbara Wetmore (Hamner Institutes for Health Sciences) for the clearance values and 398 Jacqueline Biesterbos and Nel Roeleveld (Radboud University) for product usage data. Funding 399 was provided by the University of Michigan Risk Science Center, the Dow Postdoctoral 400 Fellowship in Sustainability to SAC and the Long Range Research Initiative of the American 401 Chemistry Council.

402

403 


\section{References}

4071 Cowan-Ellsberry CE, Robison SH. Refining aggregate exposure: example using parabens. $408 \quad$ Regul Toxicol Pharmacol 2009; 55: 321-9.

4092 Gosens I, Delmaar CJE, Ter Burg W, de Heer C, Schuur AG. Aggregate exposure approaches for parabens in personal care products: a case assessment for children between 0 and 3 years old. J Expo Sci Environ Epidemiol 2014; 24: 208-14.

412

413

414

415

416

417

418

419

420

421

422

423

424

425

426

427

428

429

430

431

432

433

434

435

3 Wambaugh JF, Setzer RW, Reif DM, Gangwal S, Mitchell-Blackwood J, Arnot JA et al. High-throughput models for exposure-based chemical prioritization in the ExpoCast project. Environ Sci Technol 2013; 47: 8479-88.

4 Isaacs KK, Glen WG, Egeghy P, Goldsmith M-R, Smith L, Vallero D et al. SHEDS-HT: An Integrated Probabilistic Exposure Model for Prioritizing Exposures to Chemicals with Near-Field and Dietary Sources. Environ Sci Technol 2014; 48: 12750-9.

5 Wetmore BA, Wambaugh JF, Ferguson SS, Sochaski MA, Rotroff DM, Freeman K et al. Integration of dosimetry, exposure, and high-throughput screening data in chemical toxicity assessment. Toxicol Sci 2012; 125: 157-74.

6 Shin H-M, Ernstoff A, Arnot JA, Wetmore BA, Csiszar SA, Fantke P et al. Risk-Based High-Throughput Chemical Screening and Prioritization using Exposure Models and in Vitro Bioactivity Assays. Environ Sci Technol 2015; 49: 6760-6771.

7 Shin H-M, McKone TE, Bennett DH. Intake fraction for the indoor environment: a tool for prioritizing indoor chemical sources. Environ Sci Technol 2012; 46: 10063-72.

8 Braun JM, Just AC, Williams PL, Smith KW, Calafat AM, Hauser R. Personal care product use and urinary phthalate metabolite and paraben concentrations during pregnancy among women from a fertility clinic. J Expo Sci Environ Epidemiol; 24: 459-66.

9 Parlett LE, Calafat AM, Swan SH. Women's exposure to phthalates in relation to use of personal care products. J Expo Sci Environ Epidemiol 2013; 23: 197-206.

10 Wormuth M, Demou E, Scheringer M, Hungerbühler K. Assessments of Direct Human Exposure-The Approach of EU Risk Assessments Compared to Scenario Based Risk Assessment. Risk Anal 2007; 27: 979-990.

11 Jolliet O, Fantke P. Human Toxicity. In: Hauschild MZ, Huijbregts MAJ (eds). Life Cycle Impact Assessment. Springer Press: Dordrecht, 2015, pp 75-96.

12 Guo Y, Kannan K. A Survey of Phthalates and Parabens in Personal Care Products from the United States and Its Implications for Human Exposure. Environ Sci Technol 2013; 47: $14442-14449$. 
13 Ten Berge W. A simple dermal absorption model: Derivation and application. Chemosphere 2009; 75: 1440-1445.

14 Wilschut A, ten Berge WF, Robinson PJ, McKone TE. Estimating skin permeation. The validation of five mathematical skin permeation models. Chemosphere 1995; 30: 12751296.

15 Delmaar C, Bokkers B, Ter Burg W, Schuur G. Validation of an aggregate exposure model for substances in consumer products: a case study of diethyl phthalate in personal care products. J Expo Sci Environ Epidemiol 2014. doi:10.1038/jes.2014.68.

16 Tibaldi R, ten Berge W, Drolet D. Dermal absorption of chemicals: estimation by IH SkinPerm. J Occup Environ Hyg 2014; 11: 19-31.

17 Jolliet O, Ernstoff AS, Csiszar SA, Fantke P. Defining Product Intake Fraction to Quantify and Compare Exposure to Consumer Products. Environ Sci Technol 2015; 49: 8924-31.

18 Embry MR, Bachman AN, Bell DR, Boobis AR, Cohen SM, Dellarco M et al. Risk assessment in the 21st century: roadmap and matrix. Crit Rev Toxicol 2014; 44 Suppl 3: 6-16.

19 Soni MG, Carabin IG, Burdock GA. Safety assessment of esters of p-hydroxybenzoic acid (parabens). Food Chem Toxicol 2005; 43: 985-1015.

20 Ye X, Bishop AM, Reidy JA, Needham LL, Calafat AM. Parabens as Urinary Biomarkers of Exposure in Humans. Environ Health Perspect 2006; 114: 1843-1846.

21 CDC. Centers for Disease Control and Prevention. Fourth National Report on Human Exposure to Environmental Chemicals: Updated Tables. US Department of Health and Human Services, 2014.http://www.cdc.gov/exposurereport/.

22 Calafat AM, Ye X, Wong L-Y, Bishop AM, Needham LL. Urinary concentrations of four parabens in the U.S. population: NHANES 2005-2006. Environ Health Perspect 2010; 118: $679-85$.

23 Boberg J, Taxvig C, Christiansen S, Hass U. Possible endocrine disrupting effects of parabens and their metabolites. Reprod Toxicol 2010; 30: 301-12.

24 Błędzka D, Gromadzińska J, Wąsowicz W. Parabens. From environmental studies to human health. Environ Int 2014; 67: 27-42.

25 Rosenbaum R, Huijbregts M, Henderson A, Margni M, McKone T, van de Meent D et al. USEtox human exposure and toxicity factors for comparative assessment of toxic emissions in life cycle analysis: sensitivity to key chemical properties. Int J Life Cycle Assess 2011; 16: 710-727. 
Goldsmith M-R, Grulke CM, Brooks RD, Transue TR, Tan YM, Frame a et al.

Development of a consumer product ingredient database for chemical exposure screening and prioritization. Food Chem Toxicol 2014; 65: 269-79.

\section{7}

Bremmer HJ, Prud'homme de Lodder L, van Engelen J. Cosmetics Fact Sheet. To assess the risks for the consumer. Updated version for ConsExpo 4. RIVM report 320104001/2006. National Institute for Public Health and the Environment, Bilthoven, The Netherlands, 2006.

28 Caon T, Costa ACO, de Oliveira MAL, Micke GA, Simões CMO. Evaluation of the transdermal permeation of different paraben combinations through a pig ear skin model. Int J Pharm 2010; 391: 1-6.

29 Kitagawa S, Li H, Sato S. Skin permeation of parabens in excised guinea pig dorsal skin, its modification by penetration enhancers and their relationship with n-octanol/water partition coefficients. Chem Pharm Bull (Tokyo) 1997; 45: 1354-7.

30 Nanayakkara GR, Bartlett A, Forbes B, Marriott C, Whitfield PJ, Brown MB. The effect of unsaturated fatty acids in benzyl alcohol on the percutaneous permeation of three model penetrants. Int J Pharm 2005; 301: 129-39.

31 Nicoli S, Zani F, Bilzi S, Bettini R, Santi P. Association of nicotinamide with parabens: effect on solubility, partition and transdermal permeation. Eur J Pharm Biopharm 2008; 69: 613-21.

32 Dal Pozzo A, Pastori N. Percutaneous absorption of parabens from cosmetic formulations. Int J Cosmet Sci 1996; 18: 57-66.

33 Sugibayashi K, Todo H, Oshizaka T, Owada Y. Mathematical model to predict skin concentration of drugs: toward utilization of silicone membrane to predict skin concentration of drugs as an animal testing alternative. Pharm Res 2010; 27: 134-42.

34 Loretz LJ, Api AM, Barraj LM, Burdick J, Dressler WE, Gettings SD et al. Exposure data for cosmetic products: lipstick, body lotion, and face cream. Food Chem Toxicol 2005; 43: $279-291$.

35 Loretz L, Api AM, Barraj L, Burdick J, Davis DA, Dressler W et al. Exposure data for personal care products: Hairspray, spray perfume, liquid foundation, shampoo, body wash, and solid antiperspirant. Food Chem Toxicol 2006; 44: 2008-2018.

36 Loretz LJ, Api AM, Babcock L, Barraj LM, Burdick J, Cater KC et al. Exposure data for cosmetic products: facial cleanser, hair conditioner, and eye shadow. Food Chem Toxicol 2008; 46: 1516-24.

37 Rastogi SC, Schouten A, de Kruijf N, Weijland JW. Contents of methyl-, ethyl-, propyl-, butyl- and benzylparaben in cosmetic products. Contact Dermatitis 1995; 32: 28-30. 
38 Wang L, Liao C, Liu F, Wu Q, Guo Y, Moon H-B et al. Occurrence and Human Exposure of p-Hydroxybenzoic Acid Esters (Parabens), Bisphenol A Diglycidyl Ether (BADGE), and Their Hydrolysis Products in Indoor Dust from the United States and Three East Asian Countries. Environ Sci Technol 2012; 46: 11584-11593.

39 Liao C, Liu F, Kannan K. Occurrence of and dietary exposure to parabens in foodstuffs from the United States. Environ Sci Technol 2013; 47: 3918-25.

40 Angerer J, Aylward LL, Hays SM, Heinzow B, Wilhelm M. Human biomonitoring assessment values: approaches and data requirements. Int J Hyg Environ Health 2011; 214: $348-60$.

41 Søeborg T, Frederiksen H, Andersson A-M. Considerations for estimating daily intake values of nonpersistent environmental endocrine disruptors based on urinary biomonitoring data. Reproduction 2014; 147: 455-63.

42 Kirman CR, Aylward LL, Wetmore BA, Thomas RS, Sochaski M, Ferguson SS et al. Quantitative Property-Property Relationship for Screening-Level Prediction of Intrinsic Clearance: A Tool for Exposure Modeling for High-Throughput Toxicity Screening Data. Appl Vitr Toxicol 2015; 1: 140-146.

43 Wetmore BA, Wambaugh JF, Allen B, Ferguson SS, Sochaski MA, Setzer RW et al. Incorporating High-Throughput Exposure Predictions with Dosimetry-Adjusted In Vitro Bioactivity to Inform Chemical Toxicity Testing. Toxicol Sci 2015. doi:10.1093/toxsci/kfv171.

44 Weschler CJ, Nazaroff WW. Dermal uptake of organic vapors commonly found in indoor air. Environ Sci Technol 2014; 48: 1230-7.

45 El Hussein S, Muret P, Berard M, Makki S, Humbert P. Assessment of principal parabens used in cosmetics after their passage through human epidermis-dermis layers (ex-vivo study). Exp Dermatol 2007; 16: 830-6.

46 Gouin T, van Egmond R, Sparham C, Hastie C, Chowdhury N. Simulated use and washoff release of decamethylcyclopentasiloxane used in anti-perspirants. Chemosphere 2013; 93: $726-34$.

47 Arnot JA, Brown TN, Wania F. Estimating screening-level organic chemical half-lives in humans. Environ Sci Technol 2014; 48: 723-30.

48 Biesterbos JWH, Dudzina T, Delmaar CJE, Bakker MI, Russel FGM, von Goetz N et al. Usage patterns of personal care products: important factors for exposure assessment. Food Chem Toxicol 2013; 55: 8-17. 
44

45
49 Manová E, von Goetz N, Keller C, Siegrist M, Hungerbühler K. Use patterns of leave-on personal care products among Swiss-German children, adolescents, and adults. Int $J$ Environ Res Public Health 2013; 10: 2778-98.

50 US EPA. Estimation Programs Interface Suite ${ }^{\mathrm{TM}}$ for Microsoft ${ }^{\circledR}$ Windows, $\mathrm{v}$ 4.11. 2012. 
Tables

\begin{tabular}{|c|c|c|c|c|c|}
\hline & MeP & EtP & PrP & BuP & Notes and Reference \\
\hline $\log K_{a w}$ & -5.6 & -6.0 & -5.2 & -4.9 & $\begin{array}{l}\text { Calculated from solubility and } \\
\text { vapor pressure, EPI Suite }{ }^{50} \text {. } \\
\text { Values listed here are at } 25^{\circ} \mathrm{C} \\
\text { and were corrected to skin } \\
\text { temperature of } 32^{\circ} \mathrm{C} \text { for use in } \\
\text { the model (see Sec. S.1). }\end{array}$ \\
\hline $\begin{array}{l}K_{\mathrm{p}}^{\mathrm{aq}}\left(\mathrm{cm} \mathrm{h}^{-1}\right) \\
\left(\text { geometric mean, } \mathrm{GSD}^{2}\right)\end{array}$ & $\begin{array}{c}0.012, \\
31\end{array}$ & $\begin{array}{c}0.009 \\
19\end{array}$ & $\begin{array}{c}0.009 \\
31\end{array}$ & $\begin{array}{c}0.023, \\
31\end{array}$ & $\begin{array}{l}\text { Lognormal distribution. See } \\
\text { Table S3 }{ }^{\mathrm{a}}\end{array}$ \\
\hline $\begin{array}{l}K_{\mathrm{p}}^{\mathrm{gas}}\left(\mathrm{cm} \mathrm{h}^{-1}\right) \\
\left.\text { (geometric mean, } \mathrm{GSD}^{2}\right)\end{array}$ & $\begin{array}{c}4200 \\
28\end{array}$ & $\begin{array}{c}10000 \\
28\end{array}$ & $\begin{array}{c}1400 \\
28\end{array}$ & $\begin{array}{c}1800 \\
28\end{array}$ & $\begin{array}{l}\text { Lognormal distribution. } \\
\text { Geometric mean calculated } \\
\text { using equation in Table } \mathrm{S} 1 . \\
\mathrm{GSD}^{2} \text { was set to the mean of } \\
K_{\mathrm{p}}^{\mathrm{aq}} \mathrm{GSD}^{2} . \text { Values are at } 25^{\circ} \mathrm{C} \\
\text { and were corrected to skin } \\
\text { temperature. }\end{array}$ \\
\hline $\begin{array}{l}f_{\mathrm{p}} \text { shampoo }(\%) \\
\text { (low }- \text { high) }\end{array}$ & $\begin{array}{c}0.01 \\
\text { to } \\
0.2 \\
\end{array}$ & $\begin{array}{c}0 \\
\text { to } \\
2 \times 10^{-4}\end{array}$ & $\begin{array}{l}1 \times 10 \\
3 \text { to } \\
0.2\end{array}$ & $\begin{array}{l}2 \times 10^{-} \\
4 \text { to } \\
0.045\end{array}$ & $\begin{array}{l}\text { Uniform distribution. See } \\
\text { Table } S 4^{\text {b }}\end{array}$ \\
\hline $\begin{array}{l}f_{\mathrm{p}} \text { body lotion }(\%) \\
\text { (low }- \text { high) }\end{array}$ & $\begin{array}{c}0.01 \\
\text { to } \\
0.29 \\
\end{array}$ & $\begin{array}{c}0.01 \\
\text { to } \\
0.2 \\
\end{array}$ & $\begin{array}{c}0.01 \\
\text { to } \\
0.2 \\
\end{array}$ & $\begin{array}{c}0 \\
\text { to } \\
0.085 \\
\end{array}$ & $\begin{array}{l}\text { Uniform distribution. See } \\
\text { Table } \mathrm{S} 4^{\mathrm{b}}\end{array}$ \\
\hline $\begin{array}{l}f_{\mathrm{p}} \text { range for all products } \\
\text { (low }- \text { high) }\end{array}$ & $8 \times 10^{-6}$ & $\begin{array}{c}0 \\
\text { to } \\
0.35\end{array}$ & $\begin{array}{c}0 \\
\text { to } \\
0.28 \\
\end{array}$ & $\begin{array}{c}0 \\
\text { to } \\
0.27\end{array}$ & $\begin{array}{l}\text { Uniform distribution. See } \\
\text { Table } \mathrm{S} 4^{\mathrm{b}}\end{array}$ \\
\hline $\begin{array}{l}M_{\mathrm{p}} \text { shampoo }\left(\mathrm{g} \mathrm{d}^{-1}\right) \\
\left(2.5^{\text {th }}-97.5^{\text {th }} \text { percentile }\right)\end{array}$ & \multicolumn{4}{|c|}{1.7 to 34} & Gamma distribution. $^{35}$ \\
\hline $\begin{array}{l}M_{\mathrm{p}} \text { body lotion }\left(\mathrm{g} \mathrm{d}^{-1}\right) \\
\left(2.5^{\text {th }}-97.5^{\text {th }} \text { percentile }\right)\end{array}$ & \multicolumn{4}{|c|}{2.5 to 21} & Gamma distribution. $^{34}$ \\
\hline $\begin{array}{l}M_{\mathrm{p}} \text { range for all products } \\
\left(\mathrm{g} \mathrm{d}^{-1}\right)\end{array}$ & \multicolumn{4}{|c|}{$\begin{array}{l}8 \times 10^{-4} \text { to } 44 \\
2.5^{\text {th }}-\max 97.5^{\text {th }} \\
\text { percentile) }\end{array}$} & ${ }^{34-36}$. See Figure S1 \\
\hline
\end{tabular}

Table 1: Summary input data for main model parameters for methyl, ethyl, propyl, and butyl

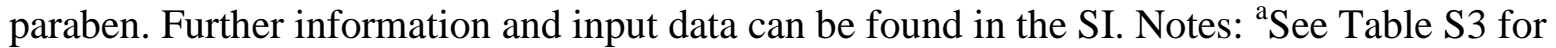
references. ${ }^{b}$ Based on data from ${ }^{1,12,37} . \mathrm{GSD}^{2}=$ geometric standard deviation squared. 
$554 \quad$ Figures
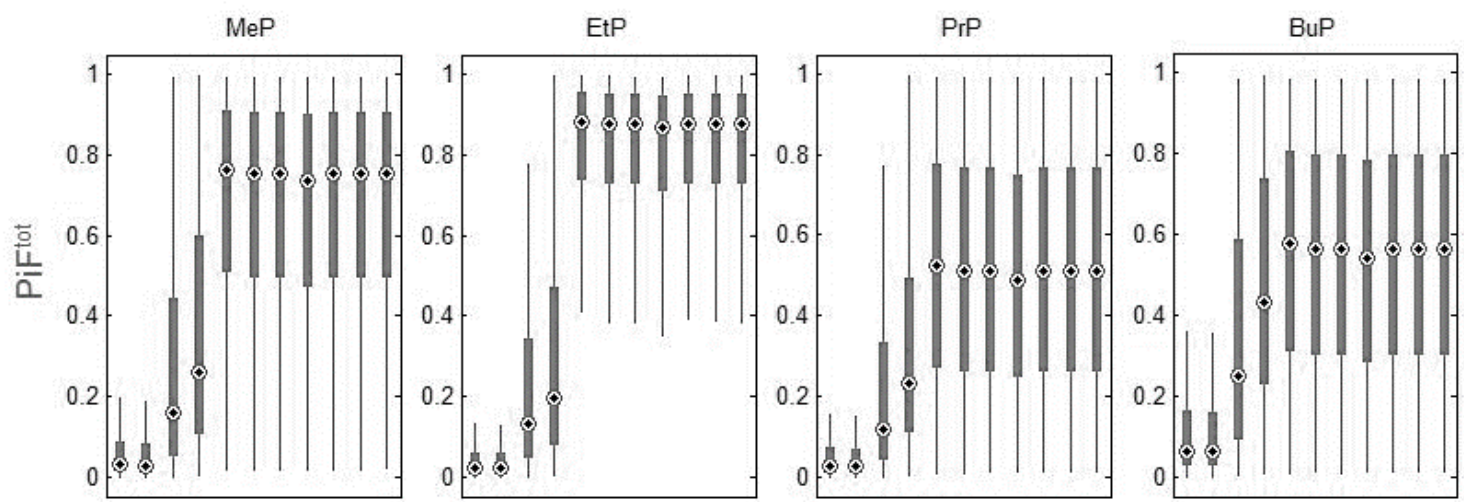

555
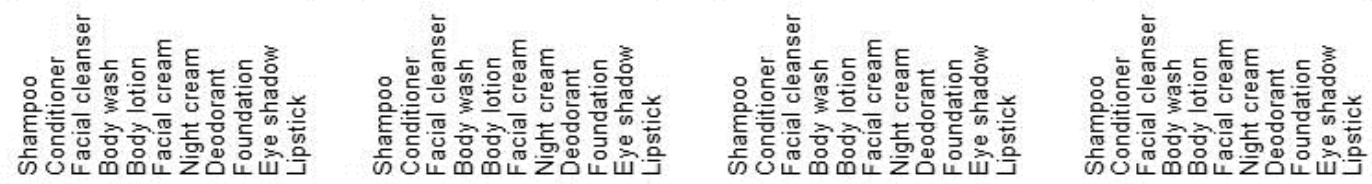

556

557

Figure 1: Modeled total product intake fraction $\left(P i F^{t o t}\right)$ for the 11 personal care products for

558 $\mathrm{MeP}, \mathrm{EtP}, \mathrm{PrP}$, and BuP (from left to right). The circles represent the median, the solid boxes 559 represent the $25^{\text {th }}$ and $75^{\text {th }}$ percentiles, and the lines represent the $2.5^{\text {th }}$ and $97.5^{\text {th }}$ percentiles of $\mathrm{PiF}^{\text {tot }}$ calculated using Monte Carlo simulation.

560 

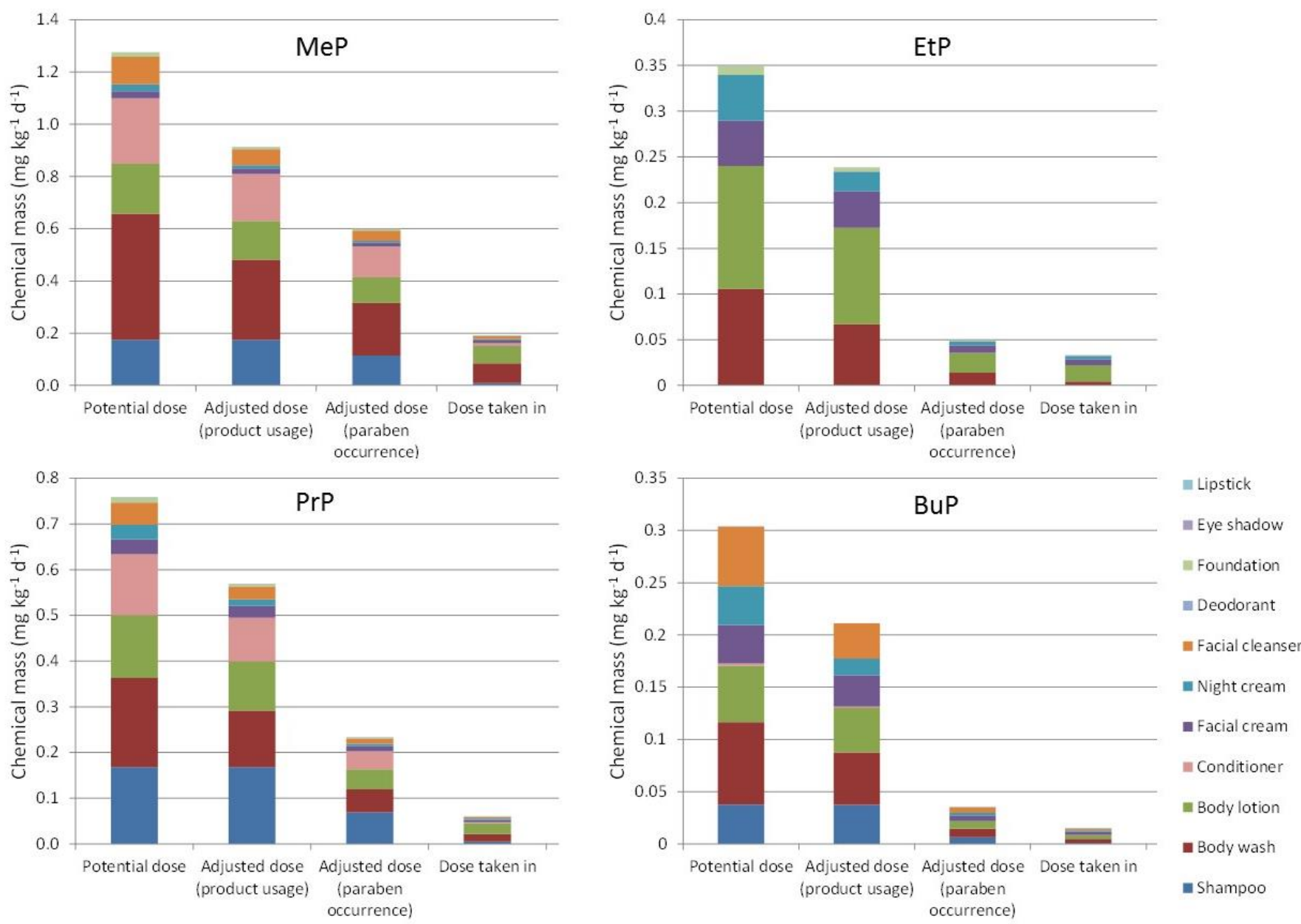

564 Figure 2: Reduction in potential applied chemical dose due to population PCP usage, paraben occurrence in products, and product intake fraction to yield the mean dose taken in for each product-chemical combination calculated by Monte Carlo simulations. The reductions were applied sequentially, thus the last column represents the dose based on all three reductions. 

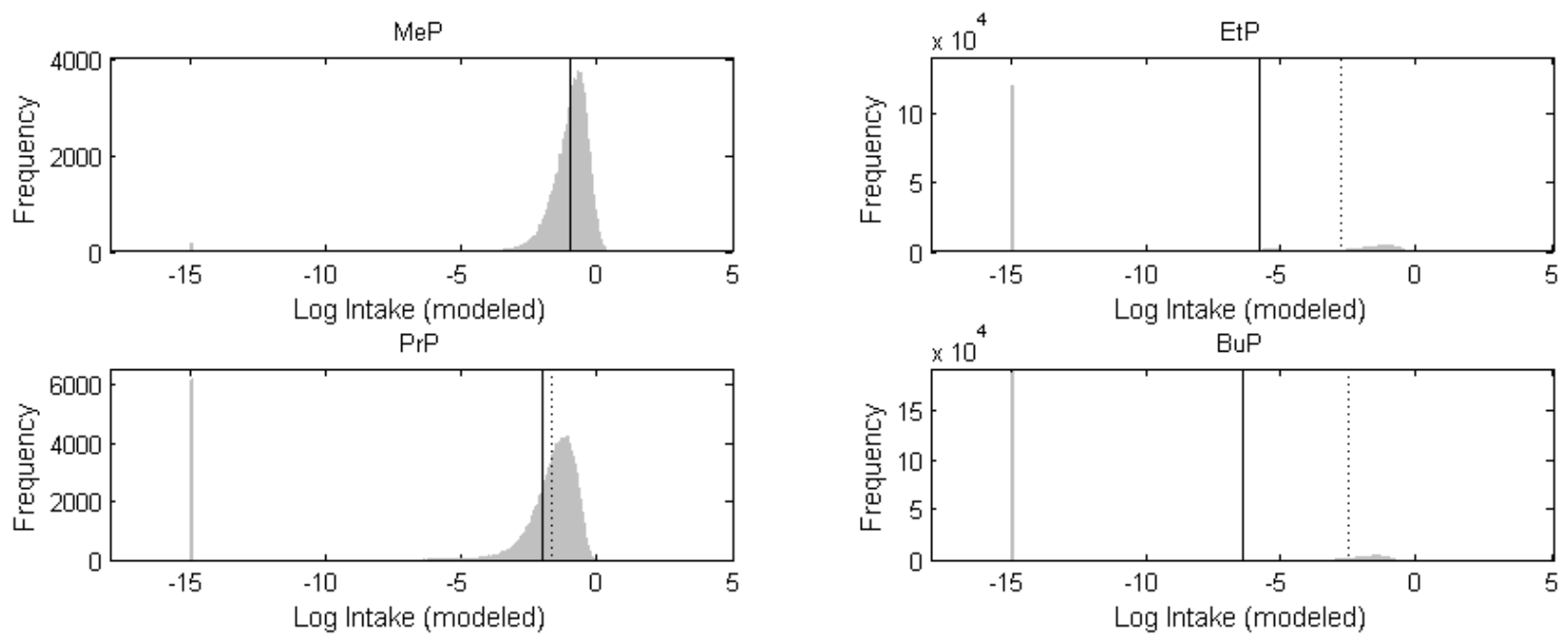

572

573

574 Figure 3: Modeled log intake $\left(\mathrm{mg} \mathrm{kg} \mathrm{d}^{-1}\right)$ distributions for the four parabens taking into account 575 probability of exposure and product co-use. The grey solid vertical lines indicate the population 576 with zero exposure (zero intakes were adjusted to a nominally low value $\left(1 \times 10^{-15}\right)$ to make them 577 visible on a log scale). The dashed line indicates the geometric mean of the exposed population 578 and the black solid line indicates the geometric mean of the entire population (with adjusted zero 579 intakes). 


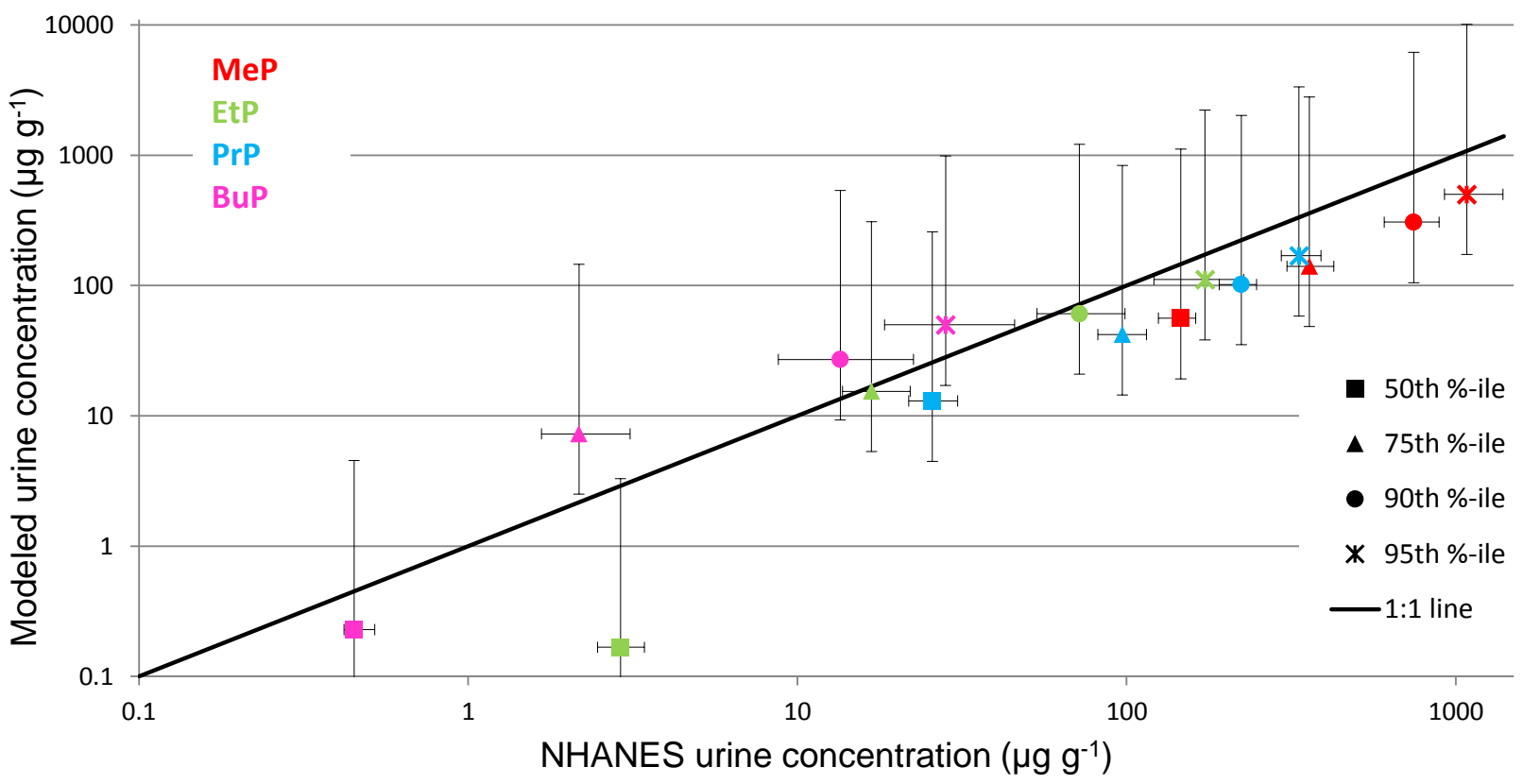

591

592

Figure 4: Modeled urine concentrations from the Monte Carlo calculations versus NHANES urine concentrations. Vertical error bars on the modeled values represent uncertainty in fraction urinary excretion and horizontal error bars on the NHANES values represent the $95^{\text {th }}$ confidence interval on each percentile. The solid line indicates perfect agreement between modeled and measured values (1:1 line). 